Revista de Psicología Vol. 31 (2), 2013 (ISSN 0254-9247)

\title{
Universidades saludables: una apuesta a una formación integral del estudiante
}

\author{
Silvia Becerra Heraud \\ Pontificia Universidad Católica del Perú $\mathbf{1}^{*}$
}

Se presenta la propuesta de universidades saludables como un enfoque de promoción de la salud viable y necesario a trabajar en las instituciones de educación superior. Se expone su definición y características básicas, los pasos necesarios para facilitar su implementación adecuada y una reseńa histórica acerca de los proyectos existentes en universidades de América y Europa. En el Perú existen varias iniciativas, entre las que destaca el trabajo realizado por el Consorcio de Universidades y, actualmente, el inicio del programa PUCP Saludable en la Pontificia Universidad Católica del Perú. Finalmente, se realiza una reseńa sobre el trabajo realizado a la fecha por este programa.

Palabras clave: universidad saludable, universidad promotora de la salud, promoción de la salud, programa PUCP Saludable

\section{Healthy universities: betting on an integral student formation}

This paper presents the proposal of healthy universities as an approach to health promotion feasible and necessary to work in higher education institutions. It exposes its definition and basic features, the steps to facilitate proper implementation and a historical review about the existing projects in universities of America and Europe. In Peru, there are several initiatives, among which the work of the Consortium of Universities and currently the start of the PUCP Healthy Program at the Pontifical Catholic University of Peru. Finally, it is done a brief review of the work implemented to date by this program.

Keywords: healthy university, health promoting university, health promotion, PUCP Healthy Program

1 Magíster en Psicología por la Pontificia Universidad Católica del Perú (PUCP), psicoterapeuta conductual-cognitiva con adolescentes y adultos, docente por horas en la Pontificia Universidad Católica del Perú - PUCP y en el Instituto Peruano de Terapia ConductualCognitiva -IPETEC. Contacto: Calle Requena 196, Lima 33, Perú; sbecerra@pucp.pe 

El concepto de salud ha adquirido diferentes connotaciones desde que la OMS en 1947 la definió como "el estado de bienestar completo en los aspectos físicos, mentales y sociales del ser humano y no solo la ausencia de enfermedades y padecimientos" (como se citó en Taylor, 2007). El sentido actual en la concepción de salud y de enfermedad ha implicado una atención a los factores psicosociales que afectan a los sistemas corporales, lo cual altera la vulnerabilidad del individuo ante los procesos patológicos. Además, este giro ha originado que se preste gran atención al concepto de estilo de vida y, específicamente, a los hábitos relacionados con la salud, como principales determinantes de la calidad del bienester psicológico y físico (Oblitas, 2010; Organización Mundial de la Salud [OMS], 2002). En este nuevo concepto de salud, se destaca que no solo son los comportamientos que las personas realizan los promotores de la enfermedad, sino que pone el acento además en el ambiente. Como Sigerist (1941; citado en Ippolito-Shepherd, 2010) afirma:

la salud se promueve mediante condiciones y estándares decentes de vida, buenas condiciones laborales, educación, cultura física, medios de descanso y recreación... salud no es solo la ausencia de enfermedad, sino algo positivo, una actitud optimista frente a la vida y la aceptación de las responsabilidades que la vida nos da... (p. 26).

Sin embargo, bajo este enfoque, el individuo cobra un especial acento al identificarse como un agente responsable de su salud.

La buena salud es uno de los recursos para el progreso personal, económico y social de un país, debido a que contribuye de manera importante a mejorar la calidad de vida en las comunidades (Morello, 2010). Las personas saludables y mejor preparadas son indispensables para el desarrollo individual y colectivo. El Banco Mundial concluyó que una mejor educación lleva a mejores resultados en salud y que una mejor salud lleva a mejores logros académicos (Banco Mundial, 1999). 
En esta línea, la propuesta de universidad saludable tiene como base un concepto de salud integral, el cual es producto de las relaciones armónicas internas y externas que cada persona logra mantener consigo mismo, con los demás y con el medio ambiente (Machado, 2001).

El fundamento para la implementación de una universidad saludable está incluido dentro de las políticas de salud pública de todo país, bajo el rubro de promoción de la salud. Este concepto implica desarrollar intervenciones que ayuden a las personas a poner en práctica conductas saludables y modificar conductas inadecuadas (Taylor, 2007). Según el Ministerio de Salud (MINSA, 2010), la promoción de la salud es "un proceso que busca desarrollar habilidades personales y generar mecanismos administrativos, organizativos y políticos que faciliten a las personas y grupos tener mayor control sobre su salud y mejorarlo" (p. 29). La promoción de la salud se lleva a cabo en muchos espacios diferentes y se parte de la base que "la salud es construida y vivida por las personas en su ambiente cotidiano, donde ellas trabajan, aprenden, juegan y aman” (Lange \& Vio, 2006, p. 9). Es por ello que las universidades tienen una fuerza potencial para influir positivamente en la vida y la salud de sus miembros. Son instituciones en donde las personas pasan una parte importante de sus vidas y pueden incidir en la formación de sus estilos de vida, llevándolos a ser personas autónomas, reflexivas, críticas, con responsabilidad frente a sí mismos y ante los demás; además los universitarios lideran innovaciones y tienen un rol modélico en la sociedad. Asimismo, las actividades principales de la universidad son la docencia y la investigación, lo que permite la incorporación del concepto de salud como una responsabilidad personal y social (Arroyo \& Rice, 2009; Lange \& Vio, 2006; Morello, 2010; Propuesta de Modelo Nacional de Universidad Saludable - PREVENIMSS, 2007).

En forma más precisa, se puede definir a una universidad saludable o una universidad promotora de salud como aquella que incorpora la promoción de la salud a su proyecto educativo y laboral, con el fin de propiciar el desarrollo humano y mejorar la calidad de vida de quienes allí estudian o trabajan y, a la vez, formarlos para que actúen como modelos o promotores de conductas saludables a nivel de sus familias, 
en sus futuros entornos laborales y en la sociedad en general (Granados, 2010b; Lange \& Vio, 2006; Machado, 2001). Según Tsouros (1998) el concepto de una universidad promotora de salud significa mucho más que dirigir una educación en salud y una promoción de la salud para estudiantes y el equipo. Significa integrar la salud dentro de la cultura, procesos y políticas de la universidad. Significa comprender y lidiar con la salud de una forma diferente y desarrollar un marco de acción que combina factores como empoderamiento, diálogo, elección y participación con metas para la equidad, sostenibilidad y una vida que conduce a la salud, entornos de trabajo y aprendizaje.

Bajo los programas de universidades saludables se pueden trabajar temas como alimentación y nutrición; salud sexual y reproductiva; promoción de la salud mental, buen trato, cultura de paz y habilidades para la vida; actividad física; seguridad vial y cultura de tránsito y, finalmente, la conservación del ambiente, ecoeficiencia y salud (Lange \& Vio, 2006; MINSA, 2010). Asimismo, también es importante trabajar con otras conductas relacionadas con la salud como los hábitos del sueño y el descanso, el consumo de alcohol, tabaco y otras drogas, las conductas de autocuidado y el manejo de la recreación y del tiempo libre (Arrivillaga, Salazar \& Correa, 2003; Salazar, Varela, Lema, Tamayo \& Duarte, 2010).

Podemos proponer que, esencialmente, una universidad saludable es la que provee un ambiente saludable, favoreciendo el aprendizaje a través de sus áreas y edificaciones, zonas de recreación, bienestar universitario y medidas de seguridad. También sería la que incorpora ejes temáticos de promoción de la salud en las mallas curriculares de todas las carreras de pre-grado e impulsa cursos de post grado, diplomados y maestrías relacionados a promoción de la salud. Asimismo, fomenta la actividad física: deportes, ciclismo, caminatas, etc., como parte de los programas curriculares y extra-curriculares de la institución y las prácticas saludables de alimentación y nutrición en el comedor y los lugares de expendio de alimentos, incluyendo normas que rigen la naturaleza de la alimentación ofrecida dentro de sus instalaciones. Es importante recalcar que una universidad saludable también implica promover el cumplimiento 
de las políticas públicas como la ley 28705, Ley General para la Prevención y Control del Consumo del Tabaco: ambientes 100\% libres de humo de tabaco (en el caso del Perú), realizando campañas de sensibilización sobre el tema e, inclusive, ofreciendo programas para dejar este hábito; además realiza actividades en las que no se consumen bebidas alcohólicas. Por último, sería la que facilita el consumo de agua segura y promueve la ingesta de mínimo dos litros de agua al día e impulsa actividades de información, fomento y consejería en: alimentación y nutrición saludable, salud sexual y reproductiva, salud mental y prevención del consumo de alcohol, tabaco y otras drogas, sueño saludable, autocuidado y cuidado médico (Machado, 2001; MINSA, 2010).

Para lograr lo mencionado, autores como Arroyo y Rice (2009) y Lange y Vio (2006) sugieren cumplir con una serie de pasos y condiciones, entre las que se mencionan:

1. La designación de un comité coordinador de la iniciativa. Este comité puede dirigir el programa y reunir a otros agentes de la institución interesados que tengan la potestad de tomar decisiones y la capacidad de influencia.

2. La realización de campańas de información, concientización y sensibilización hacia los conceptos de promoción de la salud y universidad promotora de la salud. Esto permitirá que toda la población universitaria conozca sobre la iniciativa y se identifique con los objetivos propuestos, ayudando a su consecución.

3. El desarrollo del análisis de la situación social y de salud de los integrantes de la comunidad universitaria (estudiantes, profesores, personal no docente, etc.). La información sobre la línea base permitirá identificar los problemas prioritarios a trabajar y también poder contar con un referente al evaluar la efectividad del programa.

4. El desarrollo del perfil de los programas, proyectos, servicios y actividades universitarias que se relacionan con la promoción de la salud y aquellos que podrían vincularse más directamente al quehacer de las universidades promotoras de la salud. Es importante un trabajo sistematizado y organizado, bajo un modelo de marco lógico por ejemplo, que garantice una implementación adecuada. 
5. La presentación pública en la universidad de los resultados obtenidos en los puntos 3 y 4 . Hacer conocer a toda la comunidad universitaria de la información obtenida y del programa a realizar permitirá abrir puertas y coordinar esfuerzos de las diferentes instancias.

6. El desarrollo del plan estratégico institucional para impulsar la iniciativa. Se hace necesario incorporar las metas planteadas en el programa dentro de los objetivos generales de la institución, esto le dará sustento y validez.

7. La obtención de un compromiso institucional con la implantación del plan estratégico y con la asignación de apoyo económico a la iniciativa.

8. El desarrollo de la programación de las acciones y actividades para el año académico. Es importante además conocer las actividades que se vienen realizando con regularidad para poder articularlo con las actividades propuestas en el programa.

9. La definición de criterios e indicadores de medición y mecanismos de evaluación de la efectividad. Este es un criterio relevante que permitirá la permanencia del programa y el mayor apoyo a la luz de los resultados obtenidos.

10. La divulgación y publicación de los resultados de las acciones de creación, las acciones programáticas, de investigación y de evaluación vinculadas con las universidades promotoras de la salud.

Es importante mencionar que los expertos recomiendan intervenir en los aspectos organizacionales de la institución para promover una universidad saludable y así se hace crucial tomar en cuenta cinco ámbitos en los que debe integrarse una estrategia de promoción de la salud (Lange \& Vio, 2006; Muñoz, Cabieses, Contreras \& Zuzulich, 2010). Estos implican el incidir en las políticas universitarias, es decir, los objetivos deben formar parte del plan estratégico de las universidades, si es necesario modificando reglamentos, asignando recursos económicos, etc. Además, se hace importante que los estamentos responsables desarrollen una estructura organizacional que sea capaz de proponer y ejecutar articuladamente las acciones que se propongan. A nivel de los procesos se sugiere constituir un grupo interdisciplinario que 
sensibilice y promueva los lineamientos de un programa de universidad saludable. También se propone que los temas de promoción de salud deben formar parte de la malla curricular de todas las carreras y cursos de posgrado. Finalmente, se sugiere atender y modificar al ambiente físico para que este sirva de incentivo y facilite el vivir saludablemente.

\section{Antecedentes de los proyectos de universidades saludables}

La preocupación por la salud siempre ha sido una constante en la historia de la humanidad y se puede observar que varios eventos y documentos son la antesala de lo que actualmente podemos denominar como el movimiento de universidades saludables. Es importante mencionar dos específicamente: la Declaración de Alma-Ata, Salud para Todos (OMS y Unicef, 1978) y la Carta de Ottawa para la Promoción de la Salud. La primera fue elaborada durante la Conferencia Internacional de Atención Primaria de la Salud que se realizó en setiembre de 1978 en la ciudad de Alma-Ata, Kazajistán; en ella se declara a la salud como un derecho humano y como el objetivo a alcanzar en el año 2000. La segunda fue producto de la Primera Conferencia Internacional sobre promoción de la salud realizada en Ottawa, Canadá el 21 de noviembre de 1986. En este documento se ofrece una nueva concepción de la salud pública en el mundo y se establecen los objetivos de la salud para todos (OMS, 1986; Organización Panamericana de la Salud [OPS], 1978).

A raíz de estas y otras iniciativas es que se comienza a trabajar bajo un enfoque de "entornos saludables", lo cual implica preocuparse por todo el ambiente que conforma a una comunidad determinada y no por el individuo aislado únicamente; este enfoque aborda la salud como un producto de interdependencia entre individuos y su ambiente social (Shain \& Kramer, 2004). La oficina Regional de la Organización Mundial de la Salud lidera en Europa a partir de la década de 1980 iniciativas bajo este enfoque, aplicándolo en ciudades y autoridades locales, escuelas, universidades, organizaciones profesionales, hospitales y otros escenarios. El propósito ha sido impulsar la promoción de la 
salud a través de la estrategia de intervención a poblaciones con énfasis en el comportamiento de los individuos y las comunidades en riesgo (Kickbusch, 2003, como se citó en Arroyo \& Rice, 2009).

En América Latina los proyectos de entornos saludables cobran fuerza recién a mediados de la década de 1990, logrando avances significativos vinculados a los escenarios de ciudades, comunidades, municipios, lugares de trabajo y escuelas. En el ámbito universitario, a partir de 1996 se formalizan redes académicas-profesionales que impulsan acciones de formación de recursos humanos en promoción de la salud y educación para la salud, y se dan los primeros pasos para formalizar el enfoque de universidades promotoras de la salud (Arroyo \& Rice, 2009). En este proceso ha sido importante el trabajo realizado por universidades tanto públicas como privadas, principalmente en países como Chile, México y Colombia; además de la organización de conferencias o congresos internacionales bajo el tema de "universidades promotoras de la salud".

Se han realizado hasta la fecha seis congresos internacionales de universidades promotoras de la salud, con sede en diferentes países iberoamericanos. El I Congreso Internacional de Universidades Promotoras de la Salud se desarrolló en Chile en el 2003 y allí se dio a conocer la estrategia para que las universidades contribuyeran a la promoción de estilos de vida saludables. El II Congreso Internacional que se realizó en Canadá en el 2005 tuvo como producto la Carta de Edmonton, la cual recoge la declaración de los principios y metas de las universidades promotoras de la salud, convirtiéndose en una guía para la puesta en marcha de estos proyectos. En el 2007 en Ciudad de Juárez, México tuvo lugar el III Congreso Internacional, durante el cual se institucionaliza la Red Iberoamericana de Universidades Promotoras de la Salud - RIUPS (Arroyo \& Rice, 2009; Muñoz et al., 2010).

El IV Congreso Internacional de Universidades Promotoras de la Salud realizado en España en 2009 estuvo organizado por la Universidad Pública de Navarra; en él se aprueba un documento conocido como la Declaración de Pamplona-Iruña que establece los valores y principios del movimiento de universidades promotoras de la salud (Universidad Pública de Navarra, 2013). El V Congreso Internacional y I Congreso 
Nacional de Universidades Promotoras de la Salud se realizó en Costa Rica en 2011; en este se establece la Declaración de Costa Rica, en la cual las universidades que conforman la RIUPS ratifican su compromiso con el avance, fortalecimiento y articulación del movimiento de universidades promotoras de la salud y con otros compromisos que buscan impulsar la promoción de la salud como estrategia fundamental (Declaración de Costa Rica, 2011). Finalmente en marzo de 2013 se realizó el VI Congreso Internacional de Universidades Promotoras de la Salud y la IV Conferencia Puertorriqueña de Salud Pública en San Juan, Puerto Rico. Durante ella se realizó una sesión especial para consensuar criterios orientadores y evaluar el proceso de certificación nacional e internacional de las instituciones de educación superior promotoras de la salud que integran las redes nacionales (Granados, junio, 2013). Está programado que el próximo congreso se realice en 2015 con sede en el Perú (Arroyo, 2012).

\section{Propuestas existentes de universidades promotoras de la salud}

\section{Experiencias latinoamericanas}

A este nivel encontramos diversas propuestas de programas de universidades saludables, algunas se han trabajado de una forma más sistemática que otras. Cabe destacar el trabajo realizado en Colombia, por la Pontificia Universidad Javeriana de Colombia y en Chile, por la Pontificia Universidad Católica de Chile. En Colombia, la Pontificia Universidad Javeriana de Colombia-PUJ ha liderado la iniciativa de implementación de un programa de universidades saludables dentro de su institución desde el año 2002. Desde la vicerrectoría se implementó este programa con un enfoque participativo para que este objetivo institucional se vaya progresivamente posicionando como un asunto de todos. Se ha trabajado en base a tres etapas: conformación de un equipo asesor del programa, la implementación del programa de mantenimiento de la salud y formulación de políticas saludables sustentadas en un conocimiento de las necesidades de salud de los miembros de la 
comunidad educativa. Todo el proceso se ha organizado metodológicamente en tres ejes que se complementan: articulación y divulgación de acciones que aporten a la construcción de comunidad, mantenimiento de la salud y el eje de educación para la salud (Granados, 2010a).

En 2010 se conformó la Red Colombiana de Instituciones de Educación Superior y Universidades Promotoras de la Salud REDCUPS- con el objetivo de que las instituciones de educación superior avocadas en los proyectos de promoción de la salud en el país $y$ en el extranjero se encuentren comunicadas, se facilite las sinergias, haya un horizonte compartido, permita caracterizar las necesidades de la población al cual se dirige y se pueda potenciar los recursos con que cuenta (Granados, 2010b).

Actualmente son 66 las instituciones que conforman la red nacional de universidades promotoras de la salud en Colombia, lo cual constituye el $26.8 \%$ de todas las universidades del país (Granados, junio, 2013). Cuenta con el apoyo del Ministerio de Salud y Protección y el de Educación, quienes cumplen un rol activo y de acompañamiento respectivamente. El estado actual de esta red denota un avanzado grado de organización y ejecución de los programas de universidades saludables pues han obtenido diversos logros, como el establecimiento de un equipo coordinador compuesto por nodos regionales y coordinadores de ejes que dirigen las iniciativas, la participación de los estudiantes en los programas, la realización de investigaciones sobre el tema, la formación transversal en promoción de la salud, la realización de congresos nacionales cada dos años y la participación en congresos internacionales, la medición de la línea de base que ha permitido evaluar los avances obtenidos y la publicación de una guía nacional que rigen a las universidades promotoras de la salud (Granados, junio, 2013). Podemos apreciar que Colombia es pues, un país líder en la región en la iniciativa de trabajar de una forma integral y hacer extensiva la aplicación de un modelo de universidad promotora de la salud a muchas instituciones universitarias en su país, además de contar con varias personas comprometidas con el rol de gestor de la promoción de la salud, lideradas por la REDCUPS. 
Respecto a las iniciativas en Chile, se documenta que desde 1998 este país ha propiciado una política de estado intersectorial y participativa de la promoción de la salud, destinada a lograr estilos de vida y ambientes más saludables para la población nacional (Arroyo \& Rice, 2009). Las experiencias exitosas en esta línea son varias, siendo la más representativa la de la Pontificia Universidad Católica de Chile; además de la Universidad de Concepción (UDEC), la Universidad del Bio-Bio (UBB), la Universidad de Los Lagos, la Universidad Austral, la INTA de la Universidad de Chile, la Universidad Metropolitana de Ciencias de la Educación (UMCE), entre otras (González \& Weil, 2010). El programa de universidades saludables en la Pontificia Universidad Católica de Chile tiene cerca de 13 ańos de existencia y cuenta con una amplia experiencia en la generación, liderazgo y desarrollo permanente de acciones que promuevan estilos de vida saludables al interior de las universidades en Chile y en el mundo. Su proyecto se encuentra en la fase de desarrollo de la encuesta diagnóstica para contar con una línea base de todos los alumnos que ingresan a la universidad y el trabajo en temas referidas a tres líneas de desarrollo importantes: actividad física, alimentación saludable y entornos saludables (ambientes libres de humo de tabaco y seguridad personal). Previamente ya se ha elaborado el marco conceptual, la constitución de un equipo interdisciplinario que dirija el programa y la realización de un diagnóstico de las conductas de salud de los estudiantes de la institución. Asimismo cuenta con un servicio de salud estudiantil que trabaja las áreas de la promoción de la salud mental, salud oral, prevención cardiovascular, sexualidad responsable, prevención de cáncer de piel, prevención y control de consumo de tabaco y otras drogas, talleres de crecimiento personal, programas de prevención del suicidio y un programa de inclusión de estudiantes con necesidades especiales (Arroyo \& Rice, 2009, Muñoz et al., 2010).

También se ha creado, en este país, la Red Universitaria de Promoción y Autocuidado-RED UPRA. Esta Red trabaja desde el año 1999 desarrollando diversas actividades dirigidas hacia estudiantes de educación superior, respecto al uso y abuso de drogas legales o ilegales. Participan 45 universidades y 8 institutos profesionales de todo 
el país, en estrecha colaboración con el Consejo Nacional de Control de Estupefacientes-CONACE. Además, se creó en Chile en 2006 la Red Nacional de Universidades Promotoras de la Salud (González \& Weil, 2010) la cual cuenta con 20 universidades afiliadas en la actualidad lo que representa el 35\% de universidades del país (Granados, junio, 2013). Es importante resaltar que en 2006 se publica la "Guía para Universidades Saludables y Otras Instituciones de Educación Superior" realizada por miembros de la Pontificia Universidad Católica de Chile y la Universidad de Chile, con el auspicio de la OPS, la cual se ha transformado en un referente técnico importante y aporta al desarrollo de la iniciativa a nivel nacional e internacional (Lange \& Vio, 2006). Se menciona que se encuentra en proceso de redacción la guía orientadora para la obtención de la certificación nacional (Granados, junio, 2013). Es así que Chile se constituye como otro país líder en la implementación de programas de universidades saludables, que viene trabajando de una manera integral y se establece como un modelo a seguir por el resto de universidades de otros países de la región.

Respecto a las iniciativas para la promoción de la salud que se han realizado en Cuba, se registra que el Ministerio de Salud Pública y el Ministerio de Educación Superior han tenido un rol protagónico, pero que estas iniciativas están dispersas bajo la dirección de diferentes instituciones. Las acciones de salud universitaria parten de las orientaciones emitidas por este último ministerio y como resultado se creó el grupo de investigación y desarrollo "Universidad: Comunidad Saludable-UCOMSA", en el cual participan estudiantes y trabajadores de diferentes instituciones universitarias. Este grupo investigador muestra como resultados acreditados más importantes la constitución de grupos de investigación estudiantil en salud universitaria, cátedras estudiantiles sobre universidades promotoras de la salud, un programa integral de salud universitaria, el diseńo teórico-metodológico de una guía para las buenas prácticas en salud universitaria, cursos de capacitación sobre salud universitaria, encuentros para intercambios de experiencias, entre otros (Figueroa, 2010). Se tienen datos de que existen 38 universidades afiliadas a la red cubana de universidades 
promotoras de la salud, lo que constituye el $57.5 \%$ de las universidades del país (Granados, junio, 2013). En 2012 se actualizó la metodología utilizada por las universidades, incluyéndose el rubro de evaluación con indicadores operativos y el de ejes transversales e incorporándose, dentro del equipo coordinador de la red, a la dirección de atención primaria de salud, lo que ha permitido las alianzas y sinergias y el fortalecimiento de la extensión universitaria (Granados, junio, 2013).

En Puerto Rico, el programa de calidad de vida funciona desde 1997 como iniciativa de la Facultad de Ciencias Médicas de la Universidad de Puerto Rico, con el propósito de educar a la comunidad universitaria en torno al bienestar pleno, la prevención del consumo de alcohol y drogas, la seguridad y el crimen, el hostigamiento sexual y las agresiones sexuales. Las políticas de implementación de comportamientos saludables responden a las funciones de la oficina de calidad de vida, articulado con el trabajo docente, de investigación y proyección de la universidad, así como con la participación activa de los estudiantes para promover la calidad de vida universitaria mediante proyectos de prevención de la salud (MINSA, 2010). Actualmente está en proceso el desarrollo y la consolidación de la red en las universidades públicas y las privadas (11 en total, que representan el $40 \%$ de las universidades del país) (Granados, junio, 2013).

En México, la Universidad Autónoma de Zacatecas-UAZ desarrolla un programa de promoción de la salud con una visión para 2020. Su objetivo general es impulsar en la comunidad universitaria la responsabilidad individual y social referente a la salud, que facilite la conformación de estilos de vida saludable que eleven su calidad de vida a través de la educación preventiva. Existen ocho propuestas de programas: de actividad física "UAZ en movimiento", de nutrición "Me veo bien, como bien, estoy bien", de salud sexual y reproductiva "Soy sexualmente responsable", de salud mental "Me siento en armonía", de adicciones "Yo ante las drogas", de salud bucal "Cuido mi sonrisa", de atención a discapacitados y de cuidado del medio ambiente "En armonía con la naturaleza". Cuenta con tres fases y proponen diversas actividades (Arroyo \& Rice, 2009; MINSA, 2010). Además se identifica que en la Universidad 
Nacional Autónoma de México se realizó un estudio proponiendo un nuevo modelo de atención integral a la salud para la universidad, en base a este se planteó reorientar el plan de trabajo institucional y generar oferta de servicios dirigidos al fomento del autocuidado de la salud de los jóvenes universitarios (López-Bárcena \& González, 2003).

En Costa Rica, la promoción de la salud se inicia en el 2005 con la designación de profesionales especialistas en el tema. Se caracteriza por llevar a cabo la atención integral en salud en la comunidad universitaria con un paradigma positivista de salud integral y vínculo permanente con el Ministerio de Salud de Costa Rica y la participación de las acciones sectoriales bajo el lema "De la Enfermedad a la Promoción de la Salud". En este país existe la prospectiva del proyecto de estilos de vida saludable de las universidades que conforman la Comisión Nacional de Rectores, siendo la Universidad de Costa Rica la pionera de esta labor con un Plan Estratégico 2009-2014 que involucra la implementación de proyectos de estilos de vida saludables en las oficinas, ambientes educativos y unidades académicas; así como ambientes libre de humo de tabaco, talleres de vida, sexualidad y afectividad para estudiantes, campañas de alimentación saludable, ferias de salud, entre otros. El trabajo está organizado en seis ejes: gestión sociopolítica, análisis de situación de salud, docencia e investigación, educación para la salud, actualización continua para el equipo de promoción y asesoría y colaboración (Arroyo \& Rice, 2009; MINSA, 2010). Actualmente son 4 las universidades públicas afiliadas a la red, lo que constituye el $100 \%$ de las universidades públicas y el $7 \%$ de las universidades del país (Granados, junio, 2013). Ya han iniciado la elaboración de una guía de universidades públicas promotoras de la salud en las que se están definiendo algunos principios orientadores que cada universidad debe desarrollar (Granados, junio, 2013).

En Ecuador, la Escuela Superior Politécnica de ChimborazoESPOCH inició la implementación de promoción de la salud a partir del año 2002, mediante la propuesta de prevención de factores de riesgo para la salud en la comunidad politécnica llevada a cabo por la Facultad de Salud Pública. Sus objetivos son: promover la salud integral y mejorar las condiciones de salud de la comunidad politécnica, 
convertir a la ESPOCH en un espacio saludable, ser una politécnica promotora de la salud y favorecer la incorporación de estrategias para lograr estilos de vida saludables en las personas que integran la comunidad politécnica. Se ha realizado un diagnóstico situacional de factores de riesgo para la salud, estudios sobre comportamientos saludables en sexualidad, respuesta frente a los riesgos y desastres y consumo de alcohol en los trabajadores de la universidad. Esto ha dado como resultado la implementación de la politécnica saludable en la generación de entornos saludables, proyectos de prevención del VIH-SIDA, plan de contingencias para la prevención de desastres naturales y fortalecimiento de capacidades institucionales, programa de información y educación mediante la publicación de boletines, proyectos de ambientes libre de humo de tabaco, entre otros (Arroyo \& Rice, 2009; MINSA, 2010).

En Argentina, el Plan Nacional Argentina Saludable comenzó en el ańo 2009 con una serie de capacitaciones para lograr universidades saludables conjuntamente con la Secretaría de Políticas Universitarias del Ministerio de Educación de la Nación y el Comité Técnico del Deporte Universitario Argentino - CTDUA con el apoyo de la Organización Panamericana de la Salud y la Red Argentina de Actividad Física (RAAF). Se tiene información que a la fecha han realizado talleres de universidades saludables en Jujuy, San Juan, Neuquén y Ushuaia, en los cuales han participado más de 20 universidades con 200 jóvenes y 60 funcionarios. La Universidad Autónoma de Entre Rios también está difundiendo la iniciativa pública del programa de prevención y promoción de la salud "Cuidate MÁS" del Gobierno de Entre Ríos y se plantea que se tiene intenciones de desarrollar una guía de universidades saludables (Morello, 2010). Asimismo, la Universidad del Norte Santo Tomás de Aquino-UNSTA en Tucumán ha incorporado las acciones de promoción de la salud a partir de 2008 y está llevando a cabo programas para el no consumo de tabaco, formación de líderes promotores de la salud, por una alimentación saludable, entre otros (MINSA, 2010). Por otro lado, la Universidad Nacional de Tucumán también está realizando actividades de promoción de la salud a cargo de la Secretaría de Bienestar Universitario; esta tiene como objetivo 
prioritario mejorar el nivel de salud integral y bienestar de la población estudiantil mediante estrategias de promoción y prevención (Universidad Nacional de Tucumán, 2013).

En el Perú, las políticas de promoción de la salud y de universidades saludables las establece el Ministerio de Salud-MINSA con el propósito de contribuir a mejorar la calidad de vida de la población (Alayo et al., 2013). Es así que en 2010 el MINSA aprobó el documento técnico "Promoviendo Universidades Saludables" por medio de la resolución ministerial $\mathrm{N}^{\circ}$ 968-2010 dirigido a universidades e institutos de educación superior (MINSA, 2010). Actualmente el MINSA está estableciendo alianzas con las universidades para orientar los esfuerzos de las universidades en la implementación de la promoción de la salud y la disminución de enfermedades transmisibles (Alayo et al., 2013).

En este país hay varias iniciativas de incorporar algunos conceptos manejados por las universidades promotoras de la salud dentro de sus lineamientos universitarios. Como iniciativa más representativa es relevante mencionar la liderada por el Consorcio de Universidades, la cual está conformadas por la Pontificia Universidad Católica del Perú, la Universidad Peruana Cayetano Heredia, la Universidad de Lima y la Universidad del Pacifico. Desde 1996 este consorcio, en el marco de la responsabilidad social, viene organizando una serie de eventos y actividades dirigidas a propiciar ambientes libres de humo de tabaco, alimentación saludable, actividad física, educación sexual, conservación del ambiente, entre otros (MINSA, 2010). En junio de 2013 el Consorcio de Universidades presentó una guía para universidades saludables que busca plasmar las condiciones que permitan generar una comunidad saludable en las universidades del país (Alayo et al., 2013).

Con relación a otras iniciativas de universidades saludables en el Perú, se encuentra que la Universidad Peruana Unión tiene una Comisión de Universidad Saludable-UpeU, que tiene a su cargo la propuesta y organización de acciones como el fomento de los estilos de vida saludable en alimentación y nutrición, ambientes libres de humo de tabaco, no venta ni consumo de licores dentro y fuera de la universidad, la prevención de enfermedades, y el desarrollo de asignaturas de cultura física 
y educación para la salud. Se reporta que en la Universidad Nacional de Ingeniería se promueve una cultura de salud y la práctica de comportamientos saludables mediante diversas actividades. También realizan programas de prevención, los cuales son liderados por el Departamento Médico. En la Universidad Nacional Mayor de San Marcos, el servicio de psicología y psiquiatría de la clínica universitaria trabaja temas de promoción de la salud mental e impulsa las cafeterías saludables. La Universidad Peruana de Ciencias Aplicadas-UPC tiene un proyecto de "UPC Saludable" propuesto en marzo de 2009 por el área de nutrición y dietética de la Facultad de Ciencias de la Salud. En el plan piloto promueve la actividad física a través de un curso, incluye la iniciativa de las "pausas activas" en lo académico y la participación de la universidad en eventos de promoción de la salud (MINSA, 2010).

Finalmente es importante mencionar la Mesa de Trabajo de Universidades para la Prevención del Consumo de Drogas que se instaló en el 2000 con la asesoría técnica de la Organización No Gubernamental DEVIDA, en la cual participan varias universidades públicas y privadas del Perú. Se reporta que han realizado, bajo este tema, dos encuentros nacionales, una jornada metropolitana, un concurso de afiche y logo y talleres y/o cursos de capacitación para los miembros representantes de la universidades. Asímismo, han publicado dos documentos, en las que resalta un Manual de Aplicación en Prevención de Consumo de Drogas en Universidades (MINSA, 2010).

Como se puede apreciar, existen varias e interesantes propuestas de programas de universidades saludables en los países de Latinoamérica, con una variedad de amplitud y formalidad de los programas aplicados. Se espera que, con el tiempo, estos programas puedan ir mejorando y ampliando su cobertura a nivel de la mayoría sino de todas las instituciones de educación superior del país y de más países de la región.

\section{Experiencias a nivel mundial}

En EE.UU., podemos encontrar la iniciativa nacional liderada por la American College Health Association-ACHA que busca proveer 
apoyo, educación, comunicaciones, productos y servicios, así como promover investigación y prácticas competentes culturalmente para fortalecer la habilidad de sus miembros de promover la salud de todos los estudiantes y de la comunidad del campus. Esta institución se formó en 1920 y actualmente cuenta con 11 organizaciones regionales afiliadas que atiende a una población de cerca de 20 millones de estudiantes universitarios. Además, edita una revista científica, The Journal of American College Health (American College Health Association [ACHA], 2013). Entre las funciones que cumple la ACHA están la de publicar semestralmente un reporte acerca de la salud de los estudiantes, ofrecer planes de seguros de salud, asegurar prácticas de inmunizaciones, aplicar programas para prevenir el abuso de sustancias, implementar estrategias para mejorar la salud mental y física de los estudiantes, organizar una reunión o encuentro anual que reúne a 1800 profesionales de la salud universitaria, entre otros (ACHA, 2013).

A nivel de Europa, hay que resaltar las iniciativas realizadas por España y el Reino Unido. En las universidades españolas se han desarrollado estrategias relacionadas con la promoción de la salud, pero han estado desarticuladas y no se han mantenido en el tiempo (Pascual, Gallardo \& Corera, 2010). El proyecto de la Universidad de Alicante es la primera referente de posteriores iniciativas, aunque este no tuvo continuidad. Otras dos universidades pioneras en la implementación de este tipo de proyectos son la Universidad Pública de Navarra y la Universidad Rey Juan Carlos-URJC. La primera inicia acciones aisladas pero su interés se vio plasmado en el plan estratégico para el período 2005-2008 bajo el cual nace el Plan de Universidad Saludable. Este plan integra las actuaciones en cuatro ámbitos de intervención: entorno universitario físico y social, currículo e investigación relacionados con la promoción de la salud, oferta de servicios relacionados con este tema e información, colaboración intersectorial y participación. El plan ya cuenta con la evaluación de los resultados, del proceso y de la estructura o adecuación de los recursos, a través de métodos cuantitativos y cualitativos (Pascual et al., 2010). La URJC en Madrid cuenta con un plan de actuación 2008-2010 con cinco objetivos fundamentales: mejorar 
la calidad de vida y salud del personal docente e investigador, identificar necesidades en los estilos de vida del personal de administración y servicios, formar a los estudiantes como agentes promotores de salud, difundir el trabajo en promoción de la salud a toda la comunidad universitaria a través de una feria de la salud y conferir transversalidad a la función social de la URJC (Pascual et al., 2010).

En la web podemos encontrar que existen en España otras universidades comprometidas con la promoción de la salud, como la Universidad de Almería, la cual cuenta con una Unidad de Promoción de la Salud que organiza una serie de actividades de información, asesoramiento y terapia en fomento de la salud y la promoción de hábitos de vida saludable; asimismo también implementa cursos en esta línea y organiza actividades de divulgación y prevención (Universidad de Almería, 2013). La Universidad de Sevilla también está comprometida y realiza programas de promoción de la salud, como el Programa Forma Joven y un programa de prevención de drogas (Universidad de Sevilla, 2013). Además, en 2008 se creó la Red Española de Universidades SaludablesREUS, formada por un conjunto de instituciones comprometidas con la promoción de la salud en el entorno universitario y cuyo objetivo es reforzar el papel de las universidades como entidades promotoras de la salud y el bienestar de sus estudiantes, su personal y de la sociendad en su conjunto, liderando y apoyando procesos de cambio social. Actualmente la REUS está compuesta de 31 universidades de toda España, el Ministerio de Sanidad, Servicios Sociales e Igualdad, estructuras de salud pública de nueve comunidades autónomas, el Ministerio de Educación, Cultura y Deporte y la Conferencia de Rectores de las Universidades Españolas-CRUE (Red Española de Universidades Saludables, 2013).

Respecto a las iniciativas en el Reino Unido, la Universidad Central de Lancashire fue una de las primeras universidades en Europa en establecer una iniciativa de universidades promotoras de la salud en 1995, financiando un proyecto piloto de dos años de duración (Dooris, 2001). Esta universidad tiene el enfoque de setting approach pues busca intervenir sobre ella como un todo y no solo sobre los estilos de vida de las personas que trabajan y estudian en ella; además integró dentro de sus 
estructuras, procesos y cultura un compromiso con la salud. Para ello contrató expertos en promoción de la salud para coordinar e impulsar iniciativas de vida saludable dentro de la universidad, entre las que se incluyen actividades "visibles" y reconocidamente promotoras de una vida sana, como ferias, charlas, celebraciones, menús saludables, promoción de la actividad física, espacios limitados y regulados para fumadores, entre otras. Asimismo, se implementaron iniciativas fundamentales pero "invisibles" para la comunidad universitaria en general, como son sus cambios de políticas, estructuras y procesos que favorecen la promoción de la salud (Lange \& Vio, 2006). También en el Reino Unido, encontramos otras iniciativas antiguas, como la de la Universidad de Lancaster.

En Europa se ha creado la Red para la Promoción de la Salud en las Universidades, la cual aspira a crear un ambiente de aprendizaje y una cultura organizacional que fortalece la salud, el bienestar y las sostenibilidad de su comunidad y posibilita a las personas lograr su pleno potencial. La red la componen investigadores, expertos y administradores de universidades europeas y busca fortalecer el rol que la universidad puede jugar en la salud del individuo y de la sociedad así como un desarrollo sustentable (European Health Promoting Universities [EuroHPU], 2013).

\section{Propuesta de la Pontificia Universidad Católica del Perú-PUCP}

Desde el año 2011 se vienen realizando acciones para implementar un programa de comunidades saludables en la PUCP. Este programa está liderado por docentes del Departamento de Psicología. La iniciativa cuenta con el respaldo de la Dirección Académica de Responsabilidad Social-DARS y del Servicio de Orientación al Estudiante. Los lineamientos del programa PUCP Saludable están sistematizados en un texto inédito realizado por las responsables de este (Chau \& Tavera, 2012). En él las autoras explican los objetivos del programa, así como las acciones llevadas a cabo hasta la fecha. El programa PUCP Saludable busca convertir a esta casa de estudio en un ambiente en el que se promueve, practique y vivencie la salud en cada ámbito del 
quehacer universitario, tanto como individuos como comunidad, logrando mejorar la calidad de vida no solo de los alumnos, sino también de los docentes y del personal administrativo. Tiene los objetivos de disminuir los problemas de salud y en ese sentido contribuir con un menor índice de ausentismo y una mejor percepción de la calidad de vida durante el estudio y el trabajo. Su eje fundamental de intervención incide sobre la instalación y mantenimiento de estilos de vida saludables desde la conducta individual y colectiva. Asimismo, busca crear espacios propicios para las prácticas de salud e involucrar, en la creación y proceso, a toda la población que estudia y trabaja en la universidad.

Hasta la fecha este programa ha realizado la fase de diagnóstico, lo cual permitió establecer una línea base que servirá de referente en la medición de los resultados. Para la consecución de esta fase se realizaron diferentes actividades como la recopilación bibliográfica sobre las experiencias de comunidades saludables en el país y en Latinoamérica, el diseño y ejecución de un diagnóstico situacional sobre los principales estilos de vida de la población universitaria con el fin de obtener un perfil de las necesidades y recursos de los estudiantes y la elaboración de un informe sobre este. Para la ejecución del diagnóstico situacional se aplicó una encuesta vía virtual a 683 alumnos de toda la Universidad (encuestas completas=369); se realizaron dos entrevistas grupales por medio de la herramienta de focus group, con la participación de un grupo de alumnos representantes estudiantiles y otro grupo de estudiantes que no pertenecían a alguna agrupación política, y entrevistas a varios actores involucrados con la salud de los estudiantes universitarios que laboran en la universidad. Fruto de ello se ha logrado identificar las principales preocupaciones de la comunidad universitaria en relación con las conductas de salud, lo que servirá de insumos para la preparación del diseño del programa.

Asimismo, dentro de esta primera fase, se está realizando una campaña de sensibilización en la comunidad universitaria sobre el programa de PUCP Saludable. Esta campaña se está implementando a través de reuniones con diversas instancias que tienen cargos directivos de la PUCP con el fin de presentar el programa y que se vaya 
haciendo conocido a este nivel, así como con la publicación de material escrito relacionado con diferentes temas de la salud. Entre ellos, consejos para un estilo de vida saludable, entrevistas al equipo coordinador del programa, entrevistas a diversos personajes por temas alusivos a la conmemoración de Días Mundiales representativos de la salud y artículos relacionados como los beneficios del uso de la bicicleta, la ley sobre la prohibición del consumo de tabaco dentro del campus, entre otros. Estas publicaciones se realizan en el periódico y revista que la PUCP publica y distribuye a toda la comunidad PUCP en forma semanal. Además se ha elaborado un video sobre este programa que ha sido presentado en la bienvenida de los cachimbos (recién ingresantes) del programa de Estudios Generales Ciencias del semestre académico 2013-1 y una actividad de difusión del programa que se realizó en la bienvenida a los cachimbos de todos los programas o facultades de la Universidad también en dicho semestre académico. En una segunda fase, está en proceso la conformación de un comité técnico con distintas autoridades claves de la universidad y la elaboración final de un cronograma detallado especificando los objetivos que se cumplirán o se han cumplido hasta la fecha, las actividades sugeridas para cumplir estos objetivos, su duración, costo, metas, responsables de dichas actividades y las fuentes de verificación de los resultados obtenidos.

Además, se ha organizado la I Feria de Nutrición, con el apoyo de profesionales del Servicio Médico de la PUCP y el auspicio de diferentes empresas productoras y distribuidoras de alimentos considerados saludables. Está en proyecto llevar a cabo para el presente ańo académico una feria de salud, la realización de más grupos focales para profundizar en conductas que han salido como de riesgo en el diagnóstico inicial y la elaboración de un panel de radiación solar. Es valioso rescatar la iniciativa de la PUCP que ya puso en marcha un programa amplio y formal para convertir a su institución en una universidad promotora de la salud. Queda todavía camino que recorrer, pues si bien se han cumplido algunas de las condiciones sugeridas para lograr esto, aún están en proceso varias de ellas, así como el logro de su inserción dentro del plan estratégico de la universidad. 
Sobre la base de la revisión realizada, podemos observar que existe un interés claro y abierto por parte de las autoridades de las diferentes universidades, para complementar la formación del estudiante y que todo individuo llegue a un completo estado de bienestar, según la definición de salud propuesta por la OMS en 1947. La maquinaria para lograrlo a nivel de las instituciones de educación superior ya se ha puesto en marcha, falta consolidar más los programas iniciados, involucrando cada vez más a los diferentes actores comprometidos con la salud del estudiante y de toda la comunidad universitaria. En particular, se hace imprescindible que el mismo individuo se comprometa activamente en esta apuesta a su formación integral.

\section{Referencias}

Alayo, M., Bambarén, E., Gueiler, M., Magill, F., Sueiro, R., Valdivieso, M. \& Vera, E. (2013). Guía universidades saludables. Lima: Comisión de Comunidades Saludables del Consorcio de Universidades.

American College Health Association-ACHA. Recuperado de www. acha.org/ el día 1 de abril del 2013.

Arrivillaga, M.. Salazar, I. \& Correa, D. (2003). Creencias sobre la salud y su relación con las prácticas de riesgo o de protección en jóvenes universitarios. Colombia Médica, 34 (4) 186-195.

Arroyo, H. (2012). Movimiento iberoamericano de universidades promotoras de la salud. Escuela de Salud Pública, recinto de Ciencias Médicas, Universidad de Puerto Rico. Recuperado de: http:// conferenciapesmexico2012.com/memorias/wp-content/ themes/tema_memorias_confpes $2012 /$ downloads/ Sesiones_Simultaneas/Sesiones_Especiales/Sesion_Especial_ VIII_Jardin/Movimiento\%20Iberoamericano\%20de\%20 Universidades\%20Promotoras\%20de\%20la\%20Salud_Hiram \%20V\%20Arroyo\%20-\%20Escuela \%20de\%20Salud $\% 20$ Publica\%20-\%20Universidad\%20de\%20Puerto\%20Rico\%20\%20Puerto\%20Rico.pdf 
Arroyo, H. \& Rice, M. (Eds.) (2009). Una nueva mirada al movimiento de universidades promotoras de la salud en las Américas. Documento de trabajo desarrollado para el IV congreso internacional de universidades promotoras de la salud. Puerto Rico: Organización Panamericana de la Salud.

Banco Mundial (1999). Educational change in Latin American and the Caribbean. Social and Human Development. Washington D.C. Recuperado de: http://www.fmmeducacion.com.ar/Sisteduc/ Banco/bmeducamerica.pdf

Chau, C. \& Tavera, M. (2012). Memorias "PUCP Saludable" 2012-2. Manuscrito inédito.

Declaración de Costa Rica (2011). Recuperado de http://www.usaludable.ucr.ac.cr/modulo-congreso.html

Dooris, M. (mayo, 2001). Health promoting universities: policy and practice-a UKperspective. Prepared for discussion at CommunityCampus partnerships for health's 5th Annual Conference, San Antonio, TX. Recuperado de http://depts.washington.edu/ccph/ pdf_files/p-dooris.pdf

European Health Promoting Universities -EuroHPU. Recuperado de http://www.eurohpu.aau.dk_ el día 1 de abril del 2013.

Figueroa, D. (2010). Impacto de la salud universitaria en la educación superior: la experiencia de Cuba. En J. Ippolito-Shepherd (Comp.), Promoción de la salud. Experiencias internacionales en escuelas y universidades (pp. 269-282). Buenos Aires: Paidós.

González, R. \& Weil, J. (2010). Universidades promotoras de la salud. La experiencia en Chile. En J. Ippolito-Shepherd (Comp.) Promoción de la salud. Experiencias internacionales en escuelas y universidades (pp. 239-251). Buenos Aires: Paidós.

Granados, M. C. (2010a). Universidades promotoras de salud. Un reto que abre posibilidades para el desarrollo humano. Experiencias en la Pontificia Universidad Javeriana. En J. Ippolito-Shepherd (Comp.), Promoción de la salud. Experiencias internacionales en escuelas y universidades (pp. 283-294). Buenos Aires: Paidós. 
Granados, M. C. (2010b). Universidades saludables. Una apuesta por la promoción de la salud de los miembros de las comunidades educativas. Bogotá: Pontificia Universidad Javeriana. Recuperado de http://javeriana.edu.co/redcups/Presentacion_PUJ_\%20REDCUPS_2010.pdf

Granados, M.C. (junio, 2013). Evaluación de los procesos de fortalecimiento de las IES/universidades como promotoras de salud, redes nacionales y procesos de certificación. Ponencia presentada en el evento: presentación de la guía de universidades saludables por el Consorcio de Universidades, Lima.

Ippolito-Shepherd, J. (2010). Estrategias de promoción de la salud y desarrollo de ámbitos académicos saludables y sostenibles. En J. Ippolito-Shepherd (Comp.), Promoción de la salud. Experiencias internacionales en escuelas y universidades (pp. 23-72). Buenos Aires: Paidós.

Lange, I. \& Vio, F. (2006) Guia para Universidades Saludables y otras instituciones de educación superior. Santiago: Productora Gráfica Andros Limitadas. Recuperado de http://www.eligevivirsano.cl/ wp-content/uploads/2012/01/Guia-Universidades-Saludables_ INTAOPS.pdf

López-Bárcena, J. \& González, M. (2003). Modelo universitario de salud integral, en la Universidad Nacional Autónoma de México. Artículo especial basado en la Conferencia Magistral del Dr. Miguel E. Bustamante dictada durante el X Congreso de Investigación en Salud Pública. Cuernavaca: Instituto Nacional de Salud Pública. Recuperado de http://www.ejournal.unam.mx/ rfm/no48-6/RFM48602.pdf

Machado, J. (2001). La universidad saludable. Revista de Ciencias Humanas, 26. Recuperado de http://www.utp.edu.co/-chumanas/ revistas/revistas/rev26/machado.htm

Ministerio de Salud (2010). Documento técnico: Promoviendo universidades saludables. Lima: Ministerio de Salud. Recuperado de $\mathrm{ftp}: / / \mathrm{ftp} 2$.minsa.gob.pe/descargas/dgps/documentos/promovUniverSaludables.pdf 
Morello, P. (2010). Universidades saludables. Ponencia presentada en el evento: II Encuentro Nacional de Vigilancia, Prevención y Control de Enfermedades Crónicas No Transmisibles. Recuperado de http://www.msal.gov.ar/argentina-saludable/pdf/PRESENTACION-Uni-saludable.pdf

Muñoz, M., Cabieses, B., Contreras, A. \& Zuzulich, M.S. (2010). El desarrollo y fortalecimiento de universidades promotoras de salud. En J. Ippolito-Shepherd (Comp.), Promoción de la salud. Experiencias internacionales en escuelas y universidades (pp. 211238). Buenos Aires: Paidós.

Oblitas, L. (2010). Psicología de la salud y calidad de vida (3ra ed.). México D.F.: Cengage Learning.

Organización Mundial de la Salud - OMS (1986). Carta de Ottawa para la promoción de la salud. Recuperado de http://www1.paho. org/spanish/hpp/ottawachartersp.pdf

Organización Mundial de la Salud - OMS (2002). Informe sobre la salud en el mundo. Reducir los riesgos y promover una vida sana. Recuperado de http://www.who.int/whr/2002/en/whr02_es.pdf

Organización Mundial de la Salud - OMS y UNICEF (1978). Declaración sobre atención primaria de la salud. Rusia: Alma-Atta, septiembre 6-12.

Organización Panamericana de la Salud-OPS (1978). Declaración Alma Ata. Conferencia internacional sobre atención primaria de la salud. URSS: OPS. Recuperado de http://www1.paho.org/spanish/ dd/pin/alma-ata_declaracion.htm

Pascual, L. M., Gallardo, C. \& Corera, C. (2010). Desarrollo de universidades promotoras de la salud: la experiencia de España. En J. Ippolito-Shepherd (Comp.), Promoción de la salud. Experiencias internacionales en escuelas y universidades (pp. 253-267). Buenos Aires: Paidós.

Propuesta de modelo nacional de universidad saludable PREVENIMSS. (2007). México: Universidad de Colima. Recuperado de http:// www2.uacj.mx/universidadsaludable/memorias/documentos/Propuesta\%20de\%20Modelo\%20Nacional\%20de\%20 Universidad\%20Saludable\%20PREVENIMSS.pdf 
Red Española de Universidades Saludables - REUS. Recuperado de http://www.msssi.gob.es/profesionales/saludPublica/prevPro mocion/promocion/UniversidadesSaludables/REUS.htm el día 1 de abril del 2013.

Salazar, I., Varela, M., Lema, L., Tamayo, J. \& Duarte, C. (2010). Evaluación de las conductas de salud en jóvenes universitarios. Revista de Salud Pública, 12 (4) 599-611.

Shain, M. \& Kramer, D. (2004). Health promotion in the workplace: framing de concept; reviewing the evidence. Occupational \& Environmental Medicine, 61(7) 643-648.

Taylor, S. (2007). Psicología de la salud (6ta ed.) México D.F.: McGrawHill/Interamericana Editores, S.A.

Tsouros, A. (1998). From the healthy city to the healthy university: project development and networking. En A. Tsouros, G. Dowding, J. Thompson \& M. Dooris (Eds.), Health promoting universities. Concept, experience and framework for action (pp. 11-19). Copenhagen: World Health Organization. Regional Office for Europe. Recuperado de http://www.euro.who.int/_ data/assets/pdf_file/0012/101640/E60163.pdf

Universidad de Almería. Recuperado de http://cms.ual.es/UAL/universidad/organosgobierno/vestudiantes/estructura/upsalud/ servicios/index.htm el día 1 de abril de 2013.

Universidad de Sevilla. Recuperado de http://www.sacu.us.es/ el día 1 de abril del 2013.

Universidad Nacional de Tucumán. Recuperado de http://www.sbu. unt.edu.ar/ el día 1 de abril de 2013.

Universidad Pública de Navarra. Recuperado de http://www.fundacion.unavarra.es/universidadsaludable/index.htm el día 15 de junio de 2013.

Recibido: 01 de agosto, 2013

Aceptado: 29 de agosto, 2013 